\title{
Less-invasive perfusion techniques may improve outcome in thoracoabdominal aortic surgery
}

\author{
Theodosios Bisdas, MD, Ahmed Redwan, MD, Mathias Wilhelmi, MD, Axel Haverich, MD, \\ Christian Hagl, MD, Omke Teebken, MD, and Maximilian Pichlmaier, MA, MD
}

Objective: The study compared the short-term outcome of distal perfusion in thoracoabdominal aortic aneurysm surgery using a mini-circuit and nonocclusive femoral cannulation with a traditional setup using a roller pump and occlusive cannulation.

\begin{abstract}
Methods: A total of 120 consecutive patients undergoing thoracoabdominal aortic aneurysm repair with femorofemoral distal perfusion from 2005 to 2008 were included in this retrospective study. Perfusion was accomplished with either a mini-circuit (centrifugal pump, low priming volume, heparin coating) and nonocclusive femoral cannulation ( $\mathrm{N}=48$, group $\mathrm{A}$ ) or conventional roller pump and occlusive cannulation $(\mathrm{N}=72$, group $\mathrm{B})$. Parameters representing tissue damage or ischemia (lactate dehydrogenase, creatine kinase, myoglobin, creatinine, glomerular filtration rate) were monitored intraoperatively and postoperatively and compared.
\end{abstract}

Results: One patient from each group required renal dialysis postoperatively. Myoglobin values were similar in both groups. Creatine kinase was significantly higher in group $\mathrm{B}(P<.001)$. Hemolysis represented from lactate dehydrogenase was higher in group $\mathrm{B}(P=.005)$. Both the traditional setup and the mini-circuit achieved a return to preoperative glomerular filtration rate by postoperative day 10 with a significantly higher decrease of glomerular filtration rate within 10 days in group B.

Conclusions: The use of mini-circuits for thoracoabdominal aortic aneurysm surgery is safe. Compared with patients undergoing suprarenal clamping for abdominal aortic aneurysm, distal and organ perfusion in thoracoabdominal aortic aneurysm surgery maintain renal function independently of the technical setup. An initial decay of glomerular filtration rate on postoperative days 1 to 4 , however, seems to be related to intraoperative leg ischemia and hemolysis caused by occlusive cannulation of the femoral vessels and the roller pump. Whether this has an effect on long-term outcome remains to be seen. (J Thorac Cardiovasc Surg 2010;140:1319-24)

Earn CME credits at

http://cme.ctsnetjournals.org

The outcome after surgery for thoracoabdominal aortic aneurysm (TAAA) pathology has been vastly improved over the years by the introduction of the so-called adjuncts. ${ }^{1-7}$ These include, among others, distal and organ perfusion techniques. ${ }^{2,3,8,9}$ Although there has been much debate about the best overall concept of distal perfusion and organ protection, less attention has been paid to the individual technical setup. Generally, there are those who prefer left-sided heart bypass ${ }^{10}$ and those who use femoro-femoral bypass. ${ }^{11-13}$ The former has the benefit of a small circuitry and potentially reduced re-

\footnotetext{
From the Department of Cardiac, Thoracic, Transplantation and Vascular Surgery, Hannover Medical School, Hannover, Germany.

Disclosures: None.

Received for publication June 8, 2009; revisions received Aug 6, 2009; accepted for publication Jan 2, 2010; available ahead of print March 26, 2010.

Address for reprints: Theodosios Bisdas, MD, Hannover Medical School, Department of Cardiac, Thoracic, Transplantation and Vascular Surgery, Carl-Neuberg-Str 1, D-30625, Hannover, Germany (E-mail: Bisdas.Theodosios@MH-Hannover.de). $0022-5223 / \$ 36.00$

Copyright $(c) 2010$ by The American Association for Thoracic Surgery doi:10.1016/j.jtcvs.2010.01.012
}

quirement of heparin, but the major disadvantage is that the right heart and lung are required to provide the full blood flow and gas exchange for both the native proximal (cranial) and the artificial distal (caudal) perfusion. This requires meticulous tuning of numerous ventilatory and circulatory parameters on behalf of the anesthetist during the operation and precludes significant hypothermia for the risk of ventricular fibrillation and the surgical option of going into hypothermic arrest if arch pathology unexpectedly requires this. Femorofemoral bypass, on the other hand, requires a more aggressive anticoagulant regimen for the presence of the oxygenator and the priming volume is higher. Furthermore, both femoral artery and vein need to be accessed, but the flexibility of this setup is greater because 2 separate circuits exist; thus, the right heart and lung do not have to be unduly strained, and tuning of the overall setup is easier in a routine setup. Both techniques have the disadvantage of a vigorous jet of retrograde blood flow in the femoral artery that may dislodge luminal debris into the renal and mesenteric circulation. Although both methods yield good results as numerous publications have demonstrated, attention has recently been focused on the technical details of these setups. Thus, cannulation-dependent ischemia of the leg with subsequent loss of renal function may alter the outcome. ${ }^{14-16}$ Also, the modality of pumping (centrifugal vs roller pumps) determines hemolysis and may 


\section{Abbreviations and Acronyms \\ $\mathrm{CK}=$ creatine kinase \\ GFR $=$ glomerular filtration rate \\ $\mathrm{LDH}=$ lactate dehydrogenase \\ POD = postoperative day \\ TAAA $=$ thoracoabdominal aortic aneurysm}

change the quality of organ protection. ${ }^{13,17-19}$ Others have pointed to the potential relevance of individual pressure and flow control of organ perfusion. ${ }^{3}$

In the Department of Cardiac, Thoracic, Transplantation and Vascular Surgery, femoro-femoral bypass, with the addition of individual perfusion catheters for the visceral and renal arteries as required, has been used almost exclusively for the past 10 years. The recent advent of interventional cannulation techniques and smaller centrifugal pump setups has changed the way coronary bypass surgery is performed and prompted investigators to look at the overall setup in terms of potential benefits of these newer techniques for TAAA surgery. ${ }^{13}$ The main difference from the classic setup is the use of heparincoated, smaller foreign surfaces; small priming volumes; centrifugal pumps with intrinsic suction capability; and the option of pulsatile flow. In addition, nonocclusive cannulation techniques allow continuous perfusion of the leg past the cannula.

Thus, we hypothesized that nonocclusive cannulation of the femoral artery and the use of a small circuit setup including a centrifugal pump with pulsatile flow may lead to less hemolysis, less peripheral ischemia, and lower rates of loss of renal function than the traditional setup in TAAA repair.

\section{PATIENTS AND METHODS}

Between January 2005 and December 2008, 158 patients underwent TAAA repair. In 67 of 158 patients (42\%), distal perfusion was achieved using a mini-circuit extracorporeal circulation system connected via a nonocclusive cannulation of both femoral vessels. Eight of 67 patients $(12 \%)$ who died during the in-hospital stay and 11 of 67 patients $(16 \%)$ who exhibited severe preoperative renal failure (glomerular filtration rate [GFR] < 20) were excluded from the analysis to avoid any bias, leaving 48 patients (group A). The other 91 of 158 patients $(58 \%)$ were perfused with a traditional roller pump system and occlusive cannulation of the femoral vessels. Twelve of 91 patients $(13 \%)$ in this group died during the hospital stay and 7 of 91 patients $(8 \%)$ with severe preoperative renal failure $($ GFR $<20)$ were excluded from the analysis, leaving 72 patients to study (group B). The transition from one to the other technique intended originally for the beginning of 2006 was gradual because it depended on the availability of the mini-circuit setup and the presence of a trained technician. No other significant changes were made to the technique or technical setup, including the operating team during that period, nor were the patients specifically selected for one or the other technique. Patient demographics, comorbidities, and characteristics are listed in Table 1.

\section{Operative Technique}

The overall procedure was highly standardized. All patients were approached from the left side while in a right lateral decubitus position through 1 or 2 intercostal spaces under single lung ventilation, dividing the costal margin as required. The diaphragm was detached radially, and the distal aorta and iliac vessels were exposed strictly retroperitoneally. Staged clamping was performed with selective reinsertion of intercostal arteries in the thoracoabdominal region if back bleeding seemed insufficient and caliber was adequate. Intercostal, visceral, and renal arteries were almost exclusively reattached by an inlay technique as combinations or individually according to the specific anatomy and pathology. Thus, combined islands were, for example, avoided in young patients or patients with Marfan disease. Distal perfusion pressures were aimed at $70 \mathrm{~mm} \mathrm{Hg}$ or greater with a minimal pump flow of $1.5 \mathrm{~L} / \mathrm{min}$. Proximal pressures were kept greater than $120 \mathrm{~mm} \mathrm{Hg}$ systolic. Spinal cord drainage was performed in all patients. Selective organ perfusion was established using 1 to 4 balloon inflatable catheters that were directly connected to the arterial line of the pump setup without selective pressure or flow monitoring. During clamping the average temperature (rectal vs esophageal) was kept at approximately $33^{\circ} \mathrm{C}$, and rewarming was started immediately on declamping before all the accessory vessels were reinserted into the prosthesis.

\section{Mini-Machine}

Initially, both arterial and venous access were obtained percutaneously using a $22 \mathrm{~F} 3$-stage cannula for venous drainage and a $15 \mathrm{~F}$ or $17 \mathrm{~F}$ cannula for arterial access in the left groin. After 15 cases, we reverted to open exposure of the femoral artery through a small transverse skin incision because 3 postoperative hematomas had required surgical intervention. The cannula is presently introduced by a puncture through a small pursestring suture in the adventitia (Novalung Novaport KU-15/17F; Novalung $\mathrm{GmbH}$ Talheim, Germany). Venous access is achieved percutaneously (Edwards FemTrack 20F-24F; Edwards Lifesciences, Irvine, Calif). The tubing, arterial filter, oxygenator, and pump head of the centrifugal pump are heparin coated, and the setup requires a priming volume of $500 \mathrm{~mL}$ (Terumo ROC-Safe; Terumo Medical Corp, Somerset, NJ). Heparin dosage for this first series was set at a conservative $300 \mathrm{IE}$ per kilogram of body weight aiming for an activated clotting time of more than 200 seconds.

\section{Roller Pump}

The traditional setup consisted of a roller pump (Stöckert, Freiburg, Germany) and large cannulas (Stöckert arterial femoral 5.2-7 mm and Stöckert venous femoral V172-22/28F) inserted into the transversely opened vessels, secured by tourniquets, an oxygenator, and an arterial filter requiring 1500 $\mathrm{mL}$ priming. Flow into and from the left leg was occluded for the duration of the perfusion. Heparin was administered at 400 IE to achieve an activated clotting time of 700 seconds.

\section{Study Design and Definitions}

Because the criteria of assignment to each technique were neither random nor consecutive in time, we compared group A (2006-2008) with group B (2005-2007) in terms of comorbidities, extent of aortic repair, preoperative clinical condition, and intraoperative characteristics to exclude any undue differences that may introduce selection bias (Tables 1 and 2).

Early postoperative outcome was assessed by markers of tissue damage (creatine kinase [CK], muscle injury/ischemia, myoglobin, muscle necrosis), hemolysis [lactate dehydrogenase (LDH)], and renal function (creatinine and GFR). The extent of hemolysis was characterized by the highest level of LDH as a single postoperative value. Myoglobin and CK, as possible contributors to renal dysfunction, were measured daily for 4 postoperative days (PODs). Creatinine was analyzed at least daily for 10 PODs. The GFR was calculated using the Cockcroft-Gault equation.

The primary outcome measure was the change of GFR during the 10POD period. In addition to the individual measurements, the area under the curve of GFR plotted against time (GFR area) and the maximum change from preoperative values were assessed $\left(\mathrm{GFR}_{\max }\right)$. The difference $\left(\mathrm{GFR}_{0-10}\right)$ between preoperative GFR and GFR on POD 10 was used to characterize 
TABLE 1. Demographics and clinical data

\begin{tabular}{lcll}
\hline & $\begin{array}{c}\text { Group A } \\
(\mathbf{n = 4 8})\end{array}$ & $\begin{array}{c}\text { Group B } \\
(\mathbf{n}=\mathbf{7 2})\end{array}$ & $\boldsymbol{P}$ \\
\hline Gender & & & \\
$\quad$ Male (\%) & $34(71)$ & $41(57)$ & .07 \\
$\quad$ Female (\%) & $14(29)$ & $31(43)$ & .19 \\
Age & & & \\
$\quad$ Median (range) & $58(20-78)$ & $60(32-78)$ & .46 \\
Comorbidities & & & \\
Hypertension (\%) & $36(75)$ & $52(72)$ & .79 \\
Diabetes mellitus & & & \\
(IDDM or non-IDDM) (\%) & $4(8)$ & $7(10)$ & .89 \\
Coronary artery disease (\%) & $7(15)$ & $13(18)$ & .74 \\
COPD (\%) & $5(10)$ & $13(18)$ & .52 \\
pAOD (Fontaine St IIa) (\%) & $3(6 \%)$ & $1(1)$ & .52 \\
Crawford classification & & & \\
$\quad$ Type I & $17(35)$ & $24(33.5)$ & .56 \\
Type II & $14(29)$ & $24(33.5)$ & .79 \\
Type III & $15(31)$ & $14(19)$ & .33 \\
Type IV & $0(0)$ & $2(3)$ & .89 \\
$\quad$ Type V & $2(5)$ & $8(11)$ & .52 \\
Emergency of operation & & & \\
elective & $41(85)$ & $60(84)$ & .93 \\
urgent & $7(15)$ & $12(16)$ & .93 \\
Renal function preoperatively & & & \\
Serum creatinine mmol/L & $80(52-108)$ & $87(70-114)$ & .12 \\
$\quad$ (range) & & & \\
GFR mL/min/173 cm ${ }^{2}$ (range) & $98(59-137)$ & $100(57-143)$ & .82 \\
\hline
\end{tabular}

IDDM, Insulin-dependent diabetes; $C O P D$, chronic obstructive pulmonary disease; $p A O D$, peripheral arterial occlusive disease; $G F R$, glomerular filtration rate.

the overall change in renal function. Furthermore, the preoperative GFR was compared with the GFR on POD 4 and POD 10 for patients of the same group to establish any significant decrease or increase of renal function within each group.

\section{Statistical Analysis}

All analyses and graphs were performed with MedCalc 9.4.2.0 (MedCalc Software, Mariakerke, Belgium). Categoric variables and frequencies are presented as percentages, and continuous variables are presented as median (range). The distribution of continuous variables was determined by the Kolmogorov-Smirnov test. A $t$ test for independent variables was performed to compare all normally distributed continuous variables, as well as the $\mathrm{GFR}_{\text {area }}$, GFR $\max$, and $\mathrm{GFR}_{0-10}$ between the 2 groups. For ordinal data with 2 categories or not normally distributed continuous variables, the Mann-Whitney test was preferred. A paired $t$ test was performed to compare the mean of differences between preoperative and postoperative GFR within the same group. A $P$ value of .05 or less was considered statistically significant for individual tests, and a $P$ value of .005 or less was considered statistically significant for the serial measurements (10 days) of postoperative CK and GFR (Bonferroni correction of $\alpha$-error). Where data points were missing, the number of available observations is reported and no assumptions are made about missing data.

\section{RESULTS}

In the mortality group, which was excluded from our study population, 3 patients from group A $(6 \%)$ and 5 patients from group B $(5.4 \%)$ died during the in-hospital stay. In each group there was 1 patient who died of intraoper-
TABLE 2. Intraoperative characteristics for the two groups

\begin{tabular}{lccc}
\hline & $\begin{array}{c}\text { Group A } \\
(\mathbf{n}=\mathbf{4 9})\end{array}$ & $\begin{array}{c}\text { Group B } \\
(\mathbf{n = 1 1 2})\end{array}$ & $\boldsymbol{P}$ \\
\hline Operation time (min) & $258(133-1792)$ & $293(90-713)$ & .26 \\
Cardiopulmonary bypass (min) & $104(38-303)$ & $123(32-390)$ & .12 \\
Clamping of aorta (min) & $80(37-123)$ & $83(47-119)$ & .69 \\
Urine (mL) & $450(40-1900)$ & $445(40-2466)$ & .84 \\
Lactate (mg/dL) & $5(1,65-19)$ & $6.7(1.11-18.89) .10$ \\
Transfusion & & & \\
$\quad$ Red blood cells* (packages) & $7(0-63)$ & $7(0-61)$ & .94 \\
$\quad$ Platelets $\dagger$ (packages) & $1(0-9)$ & $1(0-10)$ & .90 \\
$\quad$ Fresh-frozen plasma $\ddagger$ (packages) & $6(0-30)$ & $6(0-32)$ & .63 \\
\hline
\end{tabular}

*Every package contains $286 \mathrm{~mL}$. †Every package contains $200 \mathrm{~mL}$. $\ddagger$ Every package contains $250 \mathrm{~mL}$.

ative heart failure; all other patients died of a sepsis-related multiple organ failure.

In our study cohort, there were 2 patients with overt renal failure requiring dialysis, 1 in each group. Of these patients, the patient in group A required permanent dialysis. Because of extreme technical difficulties during the operation, an early thrombotic occlusion of a renal artery occurred in this patient. One patient $(2 \%)$ in group $A$ and 2 patients in group B $(2 \%)$ had postoperative paraplegia $(P=.98)$.

\section{Myoglobin}

The values in both groups peaked on day 2 , showing equal distribution. There was no statistical difference between the groups in the postoperative values of myoglobin throughout the first 4 PODs. On POD 1 the mean value of myoglobin was $1546 \mathrm{U} / \mathrm{L}( \pm 745)$ and $1855 \mathrm{U} / \mathrm{L}( \pm 1260)$ for groups $\mathrm{A}$ and $\mathrm{B}$, respectively $(P=.11)$. On POD 2 the mean value was $1638 \mathrm{U} / \mathrm{L}( \pm 1191)$ and $1616 \mathrm{U} / \mathrm{L}( \pm 1359)$ in groups $\mathrm{A}$ and $\mathrm{B}$, respectively $(P=.93)$. The mean values on PODs 3 and 4 were $1276 \mathrm{U} / \mathrm{L}( \pm 1761)$ and $740 \mathrm{U} / \mathrm{L}( \pm 1136)$, respectively, for group A and $1252 \mathrm{U} / \mathrm{L}( \pm 1448)$ and 1181 $\mathrm{U} / \mathrm{L}( \pm 1461)$, respectively, for group B, without any statistical significance.

\section{Creatine Kinase}

The CK values were normally distributed and again showed a peak on POD 1. The mean (range) for each day for both groups is presented in Figure 1. A highly significant difference is seen between the 2 groups on PODs 1 $(P<.001)$ to $3(P=.001)$, with a clear trend on POD 4 $(P=.01)$. The cumulative change of $\mathrm{CK}$ during the first 4 PODs $\left(\mathrm{CK}_{\text {area }}\right)$ was significantly higher in group B (group A: $3166 \mathrm{U} / \mathrm{L}$ vs group B: $5911 \mathrm{U} / \mathrm{L}, 95 \%$ confidence interval, 1909.74-3579.73, $P<.001)$.

\section{Lactate Dehydrogenase}

The maximum value of LDH during the early postoperative course is reported (Figure 2). The values were not normally distributed. The Mann-Whitney test showed 


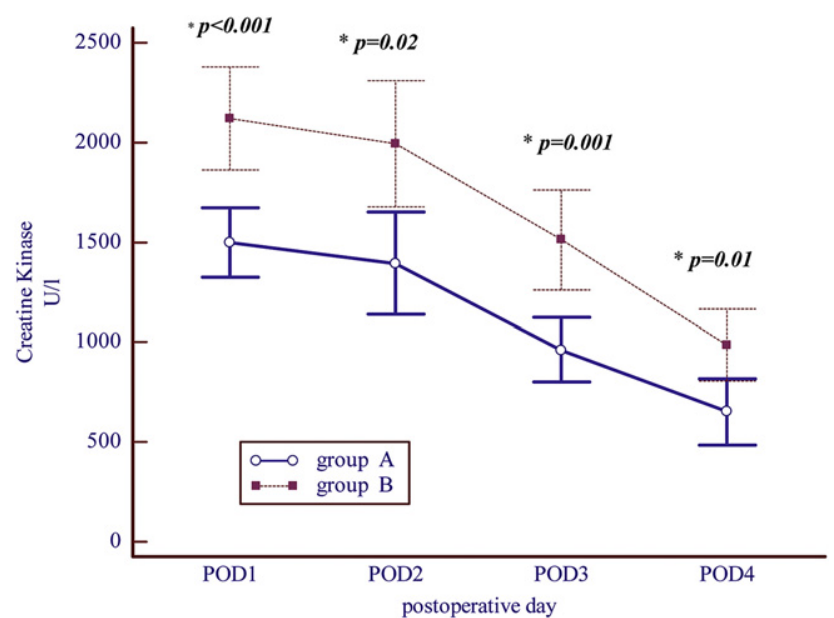

FIGURE 1. CK during postoperative course (data points represent means and $95 \%$ confidence intervals). $P O D$, Postoperative day.

a significant difference between the 2 groups, with a higher LDH increase in group B $(P=.005$, Figure 2$)$.

\section{Serum Creatinine}

The values of serum creatinine for the first 10 PODs were normally distributed for each day. The $t$ test for independent samples showed no statistically significant differences in the increase or decrease of creatinine values. By measuring the cumulative change of creatinine $\left(\mathrm{Cr}_{\text {area }}\right)$, there was also no significant difference between the groups (group A: $97.7 \pm 42 \mathrm{mmol} / \mathrm{L} / \mathrm{d}$ vs group B: $102 \pm 30.7 \mathrm{mmol} / \mathrm{L} / \mathrm{d}$, $95 \%$ confidence interval of difference: -10.3 to 19.41 , $P=.54)$.

\section{Glomerular Filtration Rate}

The GFR for each day was normally distributed. The GFR decreased significantly from preoperative values to POD 2 in each group (paired $t$ test). There was a trend, but no sta-



FIGURE 2. Comparison of maximum postoperative values of $\mathrm{LDH}$ for the 2 groups (data points represent means and $95 \%$ confidence intervals). $L D H$, Lactate dehydrogenase.
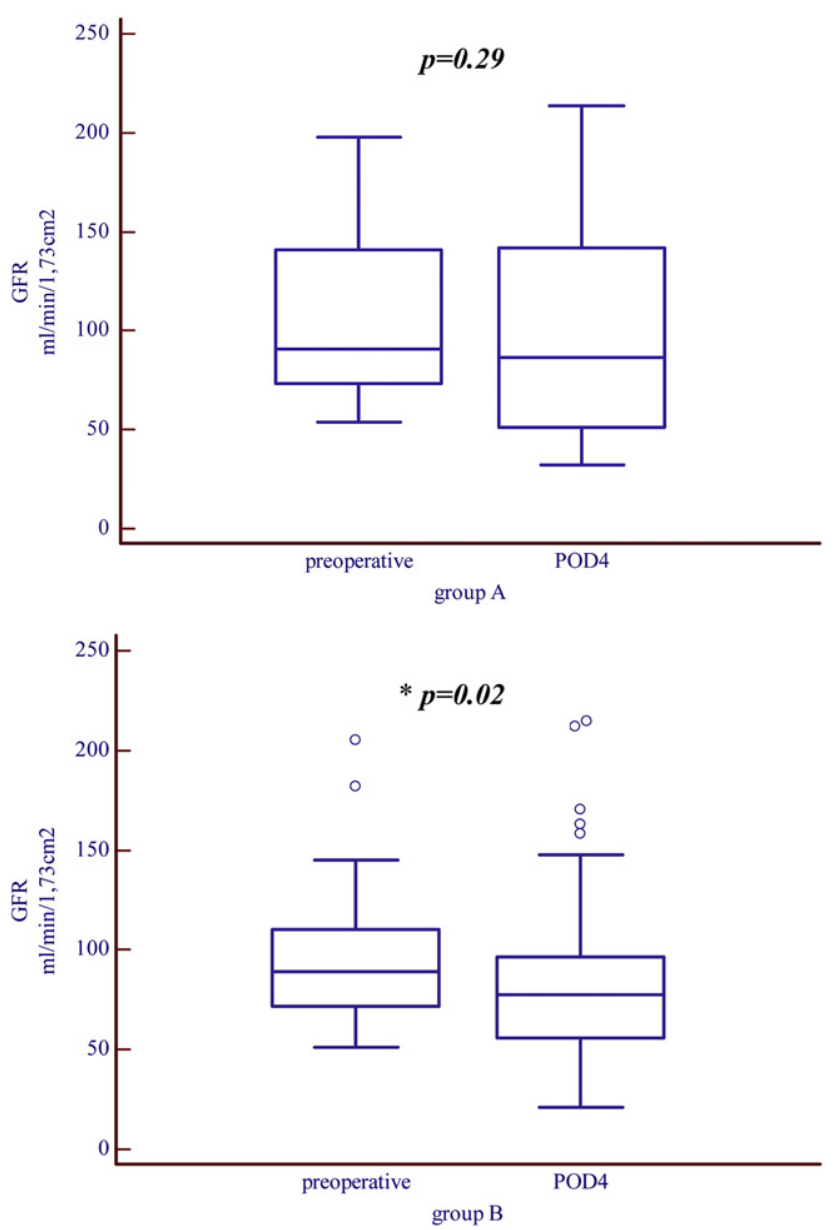

FIGURE 3. Change of GFR from preoperative to POD 4 values for each group separately (data points represent means and $95 \%$ confidence intervals). $G F R$, Glomerular filtration rate; $P O D$, postoperative day.

tistically significant difference, between the 2 groups comparing GFR for each day separately, however. The cumulative change of GFR during the initial 4- and 10POD periods, GFR $_{\text {area }}$ proved to be significant during the first 4 PODs $(P=.02)$ but not for the whole 10-POD period $(P=.74)$. Similarly, the difference between preoperative GFR and GFR on POD 10, thus the overall change in renal function, showed no statistical difference between the groups (group A: $0.59 \pm 29 \mathrm{~mL} / \mathrm{min} / 173 \mathrm{~cm}^{2}$ vs group B: $[-1.2] \pm 25, P=.73$ ). To address the size of temporary change in renal function further, we analyzed the maximum change in GFR (GFR max ) observed throughout 10 days after surgery. The $t$ test, however, reflected no significant differences between the 2 groups: group A: $(-31) \pm 26 \mathrm{~mL} /$ $\mathrm{min} / 1.73 \mathrm{~cm}^{2}$ versus group B: $(-24) \pm 22 \mathrm{~mL} / \mathrm{min} / 1.73$ $\left.\mathrm{cm}^{2}, P=.17\right)$.

In regard to the difference between the preoperative GFR and GFR on PODs 2, 4, and 10 for each group separately, the $t$ test for paired samples demonstrated a highly significant decrease on POD 2 (see above) but no significant differences for 
PODs $4(P=.07)$ and 10 in group A $(P=.90)$. However, a significant decrease of GFR was demonstrated on PODs 2 and 4 in group B $(P=.01)$, whereas a return of GFR on POD 10 to preoperative values was demonstrated $(P=.55)$ (Figure 3).

\section{DISCUSSION}

Results of TAAA surgery have improved over the years, essentially with the introduction and scientific consolidation of a number of so-called adjuncts. ${ }^{1-7}$ The aim at the time of introduction was to reduce the considerable mortality and morbidity, and especially the rate of spinal cord damage. ${ }^{20-23}$ Current results are respectable, so that more attention is paid to the aspects of morbidity, such as organ protection, especially of the kidneys. ${ }^{8,9}$ Thus, only recently attention has been drawn to the role that leg ischemia caused by occlusive cannulation of the femoral artery during distal perfusion may play in renal damage in these patients. ${ }^{14,15}$ Others have pointed to the importance of temperature on the one hand and flow and pressure control of the organ perfusion on the other hand to optimize organ protection and minimize loss of function. ${ }^{3,8,24}$ At the same time, perfusion techniques using different pumps, tubing, and cannulas, and so called mini-circuits have been established in coronary bypass surgery where reduced hemolysis and tissue damage are the focus. ${ }^{17}$

The results of this comparative analysis of a medium-sized patient population in whom occlusive traditional perfusion is contrasted with interventional mini-circuit perfusion shows first that despite clear differences in the markers for tissue ischemia on the one hand and hemolysis on the other hand, there is no clinically relevant difference in preoperative and postoperative renal function after 10 days. Both setups thus allow return of the GFR to preoperative values by that stage. This is in marked contrast with the situation in suprarenal clamping for AAA, for which there is usually a maintained loss of renal function even with short ischemic times. ${ }^{25}$ However, there is little doubt that at the same time the temporary impairment of renal function is more marked with the traditional setup compared with the mini-circuit with the additional avoidance of peripheral ischemia. The latter could be demonstrated by the significantly lower levels of markers for hemolysis and muscle tissue ischemia. The degree of leg ischemia was not specifically determined, for example, by neuromonitoring. The effect of a stenosis in the femoral artery was also caused by a large nonocclusive cannula, and therefore the relative benefit of such nonocclusive perfusion in each individual patient cannot be characterized. The beneficial effect of the nonocclusive cannulation may thus be underestimated in this study population. Furthermore, the fact that 2 parameters have been changed in the departmental policy for TAAA surgery at the same time precludes any conclusions about the relative contribution of the minicircuit and the nonocclusive distal perfusion to the observed effects. However, because there is improvement and the newer setup is, after some experience, no more cumbersome to handle and not significantly more expensive, it seems reasonable to change to a mini-circuit for TAAA surgery. The benefits of a femoro-femoral perfusion concept compared with left heart bypass are maintained because even circulatory arrest is possible with this setup.

\section{STUDY LIMITATIONS}

The authors recognize limitations of this work. The control group is to a certain extent a historical patient cohort, and it cannot be entirely excluded that other minor adjustments of the overall setup may have contributed to the results. All conceivable parameters investigated in this respect were found to be of no statistical significance. Furthermore, the team dealing with these patients and operations, and the overall policies have been remarkably constant over the period investigated. A further limitation is that this series is still small and warrants a multicenter investigation. Finally, there is no suggestion that the changes observed are of relevance in the long term; however, "noninferiority" has been shown. This is difficult to verify and may again require a multicenter approach. Similar criticism may be raised concerning the far more sophisticated methods of renal function assessment applied by others. ${ }^{8}$ Thus, the authors recognize that the parameters analyzed are generally not as specific for the assessment of the function of the different organ systems as they could be.

\section{CONCLUSIONS}

Concepts aimed to reduce tissue damage by maintaining antegrade perfusion of the extremities and to improve supply through physiologic pulsatile perfusion improve short-term outcome in TAAA surgery. Smaller pump setups for distal perfusion are safe and convey the advantages of femorofemoral bypass to the situation of TAAA surgery where minimal setups avoiding large foreign surface contact are likely to benefit the patient in this extensive surgery. Both traditional roller pump setups and mini-circuits offer adequate organ protection.

\section{References}

1. Coselli JS, Bozinovski J, Lemaire SA. Open surgical repair of 2286 thoracoabdominal aortic aneurysms. Ann Thorac Surg. 2007;83:862-4

2. Estrera AL, Miller CC III, Chen EP, Meada R, Torres RH, Porat EE, et al. Descending thoracic aortic aneurysm repair: 12-year experience using distal aortic perfusion and cerebrospinal fluid drainage. Ann Thorac Surg. 2005;80:1290-6.

3. Jacobs MJ, van Eps RG, de Jong DS, Schurink GW, Mochtar B. Prevention of renal failure in patients undergoing thoracoabdominal aortic aneurysm repair. J Vasc Surg. 2004;40:1067-73. Retraction in J Vasc Surg.2006;43:428-9.

4. Miller CC III, Porat EE, Estrera AL, Vinnerkvist AN, Huynh TT, Safi HJ. Analysis of short-term multivariate competing risks data following thoracic and thoracoabdominal aortic repair. Eur J Cardiothorac Surg. 2003;23:1023-7.

5. Safi HJ, Miller CC III, Huynh TT, Estrera AL, Porat EE, Winnerkvist AN, et al. Distal aortic perfusion and cerebrospinal fluid drainage for thoracoabdominal and descending thoracic aortic repair: ten years of organ protection. Ann Surg. 2003; 238:372-81 
6. Schepens M, Dossche K, Morshuis W, Heijmen R, van DE, Ter BH, et al. Introduction of adjuncts and their influence on changing results in 402 consecutive thoracoabdominal aortic aneurysm repairs. Eur J Cardiothorac Surg. 2004;25:701-7.

7. Weigang E, Sircar R, von SP, Hartert M, Siegenthaler MP, Luehr M, et al. Efficacy and frequency of cerebrospinal fluid drainage in operative management of thoracoabdominal aortic aneurysms. Thorac Cardiovasc Surg. 2007;55:73-8.

8. Hassoun HT, Miller CC III, Huynh TT, Estrera AL, Smith JJ, Safi HJ. Cold visceral perfusion improves early survival in patients with acute renal failure after thoracoabdominal aortic aneurysm repair. J Vasc Surg. 2004;39:506-12.

9. Köksoy C, Lemaire SA, Curling PE, Raskin SA, Schmittling ZC, Conklin LD, et al. Renal perfusion during thoracoabdominal aortic operations: cold crystalloid is superior to normothermic blood. Ann Thorac Surg. 2002;73:730-8.

10. Coselli JS. The use of left heart bypass in the repair of thoracoabdominal aortic aneurysms: current techniques and results. Semin Thorac Cardiovasc Surg. 2004;15:326-32.

11. Aomi S, Hashimoto A, Tagusari O, Nishida H, Nomura M, Kondoh I, et al. A new supportive method of aortic aneurysm surgery: centrifugal left heart bypass combined with an oxygenator and a heat exchanger. ArtifOrgans. 1996;20:700-3.

12. Coady MA, Mitchell RS. Femoro-femoral partial bypass in the treatment of thoracoabdominal aneurysms. Semin Thorac Cardiovasc Surg. 2003;15:340-4.

13. Palombo D, Valenti D, Gaggiano A, Lupo M, Borin P. Early experience with the minimal extracorporeal circulation system (MECC) during thoracoabdominal aortic aneurysm repair. Eur J Vasc Endovasc Surg. 2004;27:324-6.

14. Miller CC III, Villa MA, Achouh P, Estrera AL, Azizzadeh A, Coogan SM, et al. Intraoperative skeletal muscle ischemia contributes to risk of renal dysfunction following thoracoabdominal aortic repair. Eur J Cardiothorac Surg. 2008;33:691-4.

15. Miller CC III, Villa MA, Sutton J, Lau D, Keyhani K, Estrera AL, et al. Serum myoglobin and renal morbidity and mortality following thoracic and thoraco-abdominal aortic repair: does rhabdomyolysis play a role? Eur J Vasc Endovasc Surg. 2009;37:388-94.
16. Ouriel K. The use of an aortoiliac side-arm conduit to maintain distal perfusion during thoracoabdominal aortic aneurysm repair. J Vasc Surg. 2003;37:214-8.

17. Fromes Y, Gaillard D, Ponzio O, Chauffert M, Gerhardt MF, Deleuze P, et al. Reduction of the inflammatory response following coronary bypass grafting with total minimal extracorporeal circulation. Eur J Cardiothorac Surg. 2002;22:527-33.

18. Kofidis T, Baraki H, Singh H, Kamiya H, Winterhalter M, Didilis V, et al. The minimized extracorporeal circulation system causes less inflammation and organ damage. Perfusion. 2008;23:147-51.

19. Scott DA, Silbert BS, Doyle TJ, Blyth C, Borton MC, O'Brien JL, et al. Centrifugal versus roller head pumps for cardiopulmonary bypass: effect on early neuropsychologic outcomes after coronary artery surgery. J Cardiothorac Vasc Anesth. 2002; 16:715-22.

20. Jacobs MJ, Mess W, Mochtar B, Nijenhuis RJ, Statius van Eps RG, Schurink GW. The value of motor evoked potentials in reducing paraplegia during thoracoabdominal aneurysm repair. $J$ Vasc Surg. 2006;43:239-46.

21. Keyhani K, Miller CC III, Estrera AL, Wegryn T, Sheinbaum R, Safi HJ. Analysis of motor and somatosensory evoked potentials during thoracic and thoracoabdominal aortic aneurysm repair. J Vasc Surg. 2009;49:36-41.

22. Schurink GW, Nijenhuis RJ, Backes WH, Mess W, de Haan MW, Mochtar B, et al. Assessment of spinal cord circulation and function in endovascular treatment of thoracic aortic aneurysms. Ann Thorac Surg. 2007;83:877-81.

23. Weigang E, Hartert M, Sircar R, Samson V, Pitzer K, Genstorfer J, et al. Setup of neurophysiological monitoring with tcMEP/SSEP during thoracoabdominal aneurysm repair. Thorac Cardiovasc Surg. 2005;53:28-32.

24. Fehrenbacher JW, Hart DW, Huddleston E, Siderys H, Rice C. Optimal end-organ protection for thoracic and thoracoabdominal aortic aneurysm repair using deep hypothermic circulatory arrest. Ann Thorac Surg. 2007;83:1041-6.

25. Pichlmaier M, Hoy L, Wilhelmi M, Khaladj N, Haverich A, Teebken OE. Renal perfusion with venous blood extends the permissible suprarenal clamp time in abdominal aortic surgery. J Vasc Surg. 2008;47:1134-40. 